\title{
Investigation of particle-nucleus optical potentials for p-process nucleosynthesis
}

\author{
Jan Glorius ${ }^{* 1}$, J. Görres ${ }^{2}$, M. Knörzer ${ }^{3}$, R. Reifarth ${ }^{1}$, A. Sauerwein ${ }^{4}$, K. Sonnabend ${ }^{1}$, \\ M. Wiescher ${ }^{2}$ \\ ${ }^{1}$ Institut für angewandte Physik, Goethe Universität Frankfurt, Germany \\ ${ }^{2}$ Department of Physics, University of Notre Dame, U.S.A. \\ ${ }^{3}$ Institut für Kernphysik, Technische Universität Darmstadt, Germany \\ ${ }^{4}$ Institut für Kernphysik, Universität zu Köln, Germany \\ E-mail: glorius@iap.uni-frankfurt.de.de
}

\begin{abstract}
The majority of isotopes heavier than iron is produced via neutron-induced reactions in late stages of stars. However, the presence of a few stable, proton-rich isotopes in the solar system can only be explained with the assumption of additional proton- or $\gamma$-induced reactions. The corresponding nucleosynthesis process is usually referred to as the p-process. Starting from the remains of neutron-induced processes, the stellar composition of the isotopes is altered in explosive events by a series of photo-disintegration or proton-capture reactions. If the reaction rates needed in this network are not based on experimental data, they are typically calculated within the HauserFeshbach model. Therefore, it is mandatory to verify and improve the predictive power of these calculations studying the influence of the underlying nuclear physics. An investigation of optical particle-nucleus potentials is carried out via activation measurements of the four reactions ${ }^{165} \mathrm{Ho}(\alpha, \mathrm{n}),{ }^{166} \mathrm{Er}(\alpha, \mathrm{n}),{ }^{169} \mathrm{Tm}(\mathrm{p}, \mathrm{n})$ and ${ }^{175} \mathrm{Lu}(\mathrm{p}, \mathrm{n})$. All reactions exhibit strong sensitivities to the charged particle width of the respective entrance channel. The experimentally determined cross sections are compared to theoretical predictions of different codes using several optical potential parametrizations. Results of the investigation and a possible impact on the p-process nucleosynthesis is be presented.
\end{abstract}

XII International Symposium on Nuclei in the Cosmos

August 5-12, 2012

Cairns, Australia

\footnotetext{
* Speaker.
} 


\section{Introduction}

The stellar production mechanisms of elements above the iron region are dominated by neutron capture reactions. Moderate stellar temperatures and neutron densities are the characteristics of the slowly proceeding s process [1]. The fast component of neutron capture nucleosynthesis is the socalled $\mathrm{r}$ process taking place in exposive stellar events exhibiting extreme temperature and densities [2]. In combination, both processes are responsible for the production of nearly all heavy elements up to uranium. However, there are about 35 isotopes on the proton-rich side of stability, which cannot be produced by means of neutron capture. These isotopes, usually refered to as the p nuclei, are shielded from the capture and decay chains of $s$ and $r$ process and, hence, have to be synthesized in a distinct mechanism.

The procedure responsible for the production of the $p$ nuclei is the so-called p process $[3,4]$. Different sites and reaction mechanisms are thought to be involved. However, nowadays it is assumed that the dominant mechanism of the p process is a series of photodisintegrations of sand r-seed nuclei, which, e.g., could take place in type II supernova explosions. Initiated by $(\gamma, \mathrm{n})$ reactions the reaction flux is directed towards lower nuclear charges by competing $(\gamma, \alpha)$ and $(\gamma, \mathrm{p})$ reactions, when the neutron separation energies become higher.

This so-called $\gamma$-process reaction network consists of a large number of nuclei and reactions. For a simulation, one needs the stellar rates of all reactions involved. Because of the large amount and the often unstable reactants a measurement of all these rates is unlikely, hence, one has to rely on theoretical predictions over the whole range of the network.

In the mass region of interest, the Hauser-Feshbach model (HFM) usually is applicable due to high level densities found in the nuclei involved [5]. Beside level densities and nuclear masses, the HFM needs physics input describing the interaction of particles and nuclei. Commonly, this is treated by the optical model making use of effective interaction potentials called optical model potentials (OMP). OMPs are local or global parametrizations fitted to existing experimental data, e.g., $(\mathrm{p}, \gamma)$ and $(\mathrm{p}, \mathrm{n})$ reactions as well as $(\mathrm{p}, \mathrm{p})$ scattering for the proton OMP.

The proton OMP and the neutron OMP are thought to be understood well enough to predict cross sections with uncertainties as low as a factor of 2 . Whereas the $\alpha$-particle OMP seems to be more problematic. Reactions involving $\alpha$ particles show discrepancies between experiment and theory up to a factor of 20. This is partly due to the scarce experimental data that exists at astrophysically relevant energies in the higher mass regions. A complete set of reaction cross sections at sub-Coulomb energies would hopefully make an improvement of the predictive power possible.

The measurement of $(\alpha, \gamma)$ reactions might be useful to probe the HFM and the inverse rate, the $(\gamma, \alpha)$ reaction, can be directly used in simulations. At p process temperatures below $3 \mathrm{GK}$ the HF cross section of a $(\alpha, \gamma)$ reaction depends exclusivly on the $\alpha$ OMP [5]. However, the cross section is very small and experimentally accessible only at higher energies, where it shows sensitivity to several particle OMPs. Thus, it is difficult to identify the origin of the deviations between experiment and theory.

The aim of the present study is to get an accurate idea of the impact of the $\alpha$ OMP on the predictive power of the model and to provide reliable data to improve this specific input parameter. To achieve this $(\alpha, n)$ reactions can be measured just above the separation energy. This type of 
reaction shows a similar exclusive sensitivity to the $\alpha$ OMP at low energies as $(\alpha, \gamma)$ reactions do. But the cross section is still measurable. Consequently, a possible deviation found between experimental and calculated data can be attributed to the $\alpha$ OMP explicitly.

The conclusions in the last paragraph about the $\alpha$ OMP and $(\alpha, n)$ reactions is also valid regarding the proton OMP and $(p, n)$ reactions. Both reaction types allow an exclusive study of the $\alpha$ or proton OMP, respectively. The present investigation comprises four activation experiments on the reactions ${ }^{165} \mathrm{Ho}(\alpha, \mathrm{n}),{ }^{166} \mathrm{Er}(\alpha, \mathrm{n}),{ }^{169} \mathrm{Tm}(\mathrm{p}, \mathrm{n})$ and ${ }^{175} \mathrm{Lu}(\mathrm{p}, \mathrm{n})$.

\section{Experimental details}

The cross section measurements were carried out at the Nuclear Structure Laboratory of the University of Notre Dame in the United States. Proton and $\alpha$ beams were prepared using two different ion sources injecting into the FN tandem Van de Graaff accelerator. A terminal voltage up to $10 \mathrm{MV}$ can be provided by the machine. Proton and $\alpha$ energies between 3 to $7 \mathrm{MeV}$ and 10 to $15 \mathrm{MeV}$ were used to activate the naturally composed target materials, respectively. The beam line transmission allowed currents up to $300 \mathrm{nA}$ on target. For the analysis, the total charge collected on the Faraday cup behind the target mounting was integrated in short time intervals of 1 to 10 seconds. A negative voltage applied to an aperture in front of the Faraday cup avoided corruption of the charge measurement by deflecting secondary electrons produced by the beam.

The targets were prepared as self-supporting metallic foils. For the activation of holmium and erbium, thicknesses of around $0.8 \frac{\mathrm{mg}}{\mathrm{cm}^{2}}$ were used to achieve an energy resolution of $100 \mathrm{keV}$ to $150 \mathrm{keV}$. For proton beams, an energy resolution of 50 to $75 \mathrm{keV}$ was reached using target thicknesses of $2 \frac{\mathrm{mg}}{\mathrm{cm}^{2}}$. To monitor the target stability and to exclude loss of target material during irradiation, a silicon surface barrier detector was installed at $150^{\circ}$ with respect to the beam axis. The online Rutherford backscattering data collected in this way can also be used to remeasure target thicknesses and validate the manufacturer's statements.

The irradiations lasted between 1 hour and 3 days depending on beam energy and sample material. After the irradiation, the radioactive samples were mounted in front of a high-purity germanium (HPGe) detector to count the induced decays of the reaction products. For low-energy $\gamma$-ray emissions up to $200 \mathrm{keV}$ planar HPGe detectors with thin beryllium entrance windows where used. $\gamma$-ray energies above $200 \mathrm{keV}$ were detected with large volume coaxial HPGe detectors. Most decay schemes possess complicated cascades of $\gamma$ rays resulting in altered effective $\gamma$ intensities of the decay (summing effects). To account for this effect for each of the four reactions the sample irradiated with the highest energy was mounted in front of the detector in two geometries: at a large distance, to exclude summing effects, and at a distance very close to the detector surface to maximize efficiency. The close geometry was necessary to detect the very low reaction yields of the activations at low energy. Thus, it was possible to obtain appropriate detection efficiencies taking into account summing effects. Counting times between 1 day and 3 months were necessary to obtain sufficient statistical significance in all spectra.

\section{Analysis and results}

The number of detected $\gamma$ emissions is directly propotional to the reaction cross section: 


$$
Y_{\text {peak }}=I_{\gamma} \cdot \varepsilon\left(E_{\gamma}\right) \cdot \tau_{\text {dead }} \cdot \tau_{\text {decay }} \cdot N_{\alpha, \mathrm{p}} \cdot \mu_{\text {target }} \cdot \sigma(E),
$$

where $Y_{\text {peak }}$ is the area of a peak assigned to the decay of a reaction product, $I_{\gamma}$ the intensity of the respective $\gamma$ emission, $\varepsilon\left(E_{\gamma}\right)$ the effective detection efficiency, $\tau_{\text {dead }}$ the dead time correction, $\tau_{\text {decay }}$ the correction accounting for the decay of the unstable reaction products during irradiation, waiting time and $\gamma$ spectroscopy, $N_{\alpha, \mathrm{p}}$ the number of incident beam particles, $\mu_{\text {target }}$ the areal particle density of the target, and $\sigma(E)$ the cross section.

For comparison to predictions, the cross section is converted to an $\mathrm{S}$ factor. This is shown in comparison to theoretical predictions of the TALYS code [6] in Fig. 1 to 4 for all four reactions.

For the reactions ${ }^{165} \operatorname{Ho}(\alpha, n)$ and ${ }^{166} \operatorname{Er}(\alpha, n)$, one can see in Fig. 1 and 2 that there are several $\alpha$ OMP parametrizations available. None of them seems to be able to reproduce the experimental data satisfactorily. The TALYS standard potential according to Watanabe et al. [7] as well the potentials of McFadden and Satchler [8], Rauscher and Fröhlich [9] and Avrigeanu et al. [10] show energy dependences that do not agree with the data. Only the potential of Demetriou et al. [11, 12] seems to have an appropriate energy dependence, but still underestimates the data roughly by a factor of two.

However, the situation is different for the proton OMP as can be seen in Fig. 3 and 4. There is only one potential that is commonly applied for astrophysical calculations. The potential by Jeukenne, Lejeune and Mahaux [13] with the low-energy correction by [14] is quite successfull predicting reaction cross sections sensitive to the proton OMP. The $S$ factor of ${ }^{169} \mathrm{Tm}(\mathrm{p}, \mathrm{n})$ is overestimated by about $20 \%$. Whereas there is nearly perfect agreement with the measured $\mathrm{S}$ factor of ${ }^{175} \mathrm{Lu}(\mathrm{p}, \mathrm{n})$ deviating only by $5 \%$.

The data clearly shows that the state-of-the-art OMP parametrizations for interactions between $\alpha$ particles and nuclei are in need of improvement. Therefore, more suitable data of reactions sensitive to the $\alpha$ OMP have to be measured at energies relevant for $\mathrm{p}$ process nucleosynthesis. Especially in the mass region $A>100$, the amount of experimental data is extremely scarce indroducing uncertainties in the reaction network. Fortunately the situation is better for the proton OMP. As can be seen in literature $[16,15]$, the JLM potential is able to reliably predict cross sections in the whole $\mathrm{p}$ process network region. Nevertheless, it is desirable to further improve this parameter for a better understanding of p-nuclei nucleosynthesis.

\section{References}

[1] F. Käppeler, Prog. Part. Nucl. Phys. 43 (1999) 410.

[2] M. Arnould, S. Goriely and K. Takahashi, Phys. Rep. 450 (2007) 97.

[3] D. L. Lambert, Astron. Astroph. Rev. 3 (1992) 201.

[4] M. Arnould and S. Goriely, Phys. Rep. 384 (2003) 1.

[5] T. Rauscher, Int. J. Mod. Phys. E 20 (2011) 1071.

[6] A.J. Koning, TALYS 1.0, Proceedings of the International Conference on Nuclear Data for Science and Technology (2007).

[7] S. Watanabe, Nucl. Phys. A 713 (1958) 231. 
[8] L. McFadden and G. R. Satchler, Nucl. Phys. 84 (1966) 177.

[9] T. Rauscher, Nucl. Phys. A 719 (2003) 73.

[10] M. Avrigeanu and V. Avrigeanu, Phys. Rev. C 82 (2010) 014606.

[11] P. Demetriou, C. Grama and S. Goriely, Nucl. Phys. A 707 (2002) 141.

[12] P. Demetriou, C. Grama and S. Goriely, Nucl. Phys. A 718 (2003) 510c.

[13] J.-P. Jeukenne, A. Lejeune, C. Mahaux, Phys. Rev. C 15 (1977) 10.

[14] A. Lejeune, Phys. Rev. C 21 (1980) 1107.

[15] G. G. Kiss et al., Phys. Rev. Lett. 101 (2008) 191101.

[16] S. Harissopulos et al., Nucl. Phys. A 719 (2003) 115c.

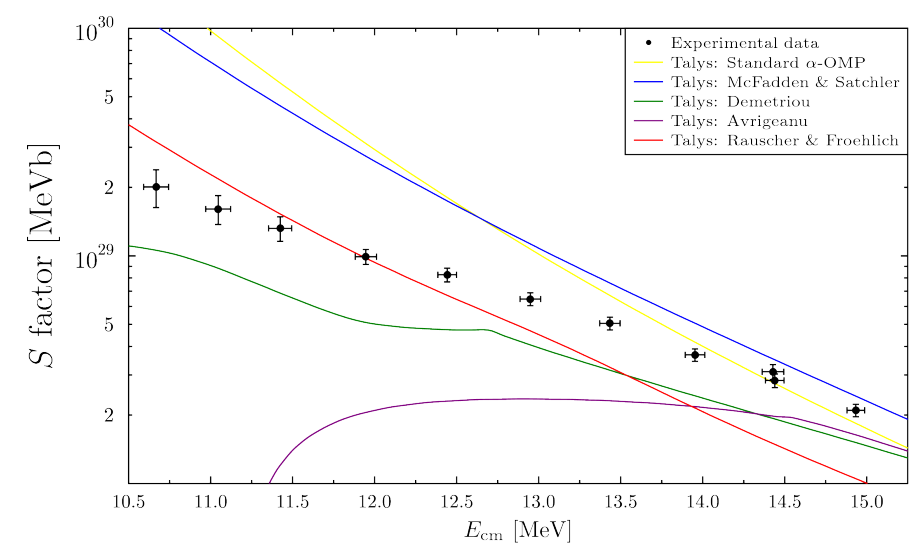

Figure 1: Experimental $S$ factor of the reaction ${ }^{165} \mathrm{Ho}(\alpha, \mathrm{n})$ compared to TALYS calculations based on different $\alpha$-OMP parametrizations. Only the potential by Demetriou et al. is able to reproduce the measured energy dependence, but still underestimates the values by a factor of 2 .

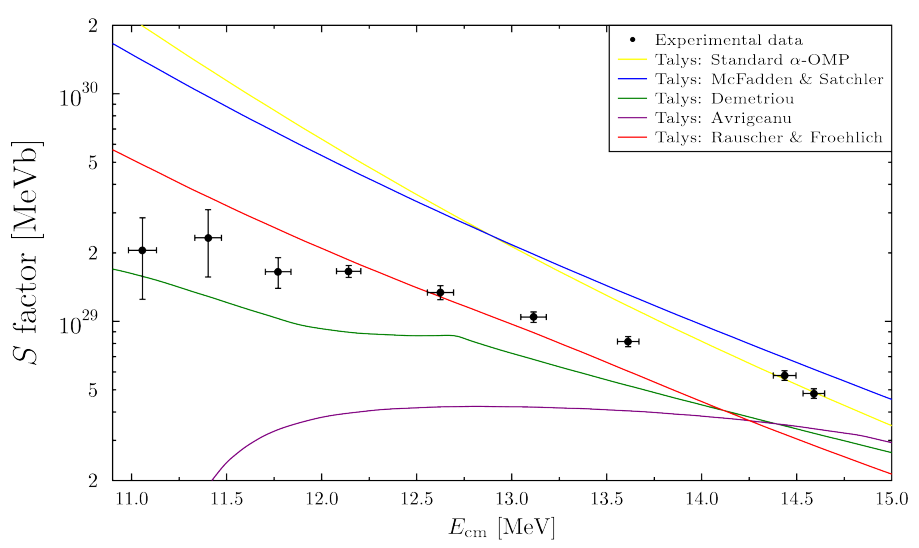

Figure 2: Experimental $S$ factor of the reaction ${ }^{166} \operatorname{Er}(\alpha, n)$ in comparisson to predictions of the TALYS code using different $\alpha$-OMP parametrizations. The potential by Demetriou et al. can reproduce the energy dependence, but the prediction is still to low by a factor 2 . 


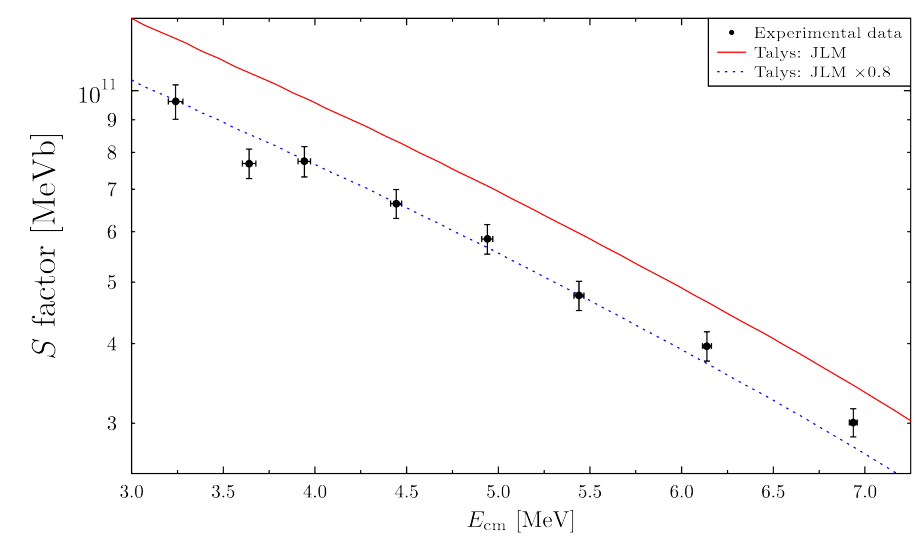

Figure 3: Experimental S factor of the reaction ${ }^{169} \mathrm{Tm}(\mathrm{p}, \mathrm{n})$ compared to the TALYS calculation based on the JLM parametrization of the proton OMP. The experimental values are overestimated by about $20 \%$.

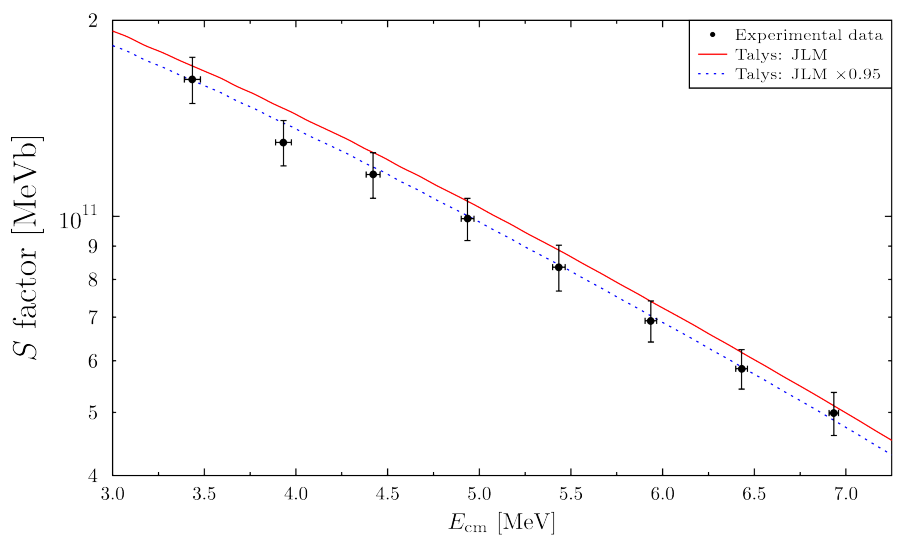

Figure 4: Experimental $\mathrm{S}$ factor of the reaction ${ }^{175} \mathrm{Lu}(\mathrm{p}, \mathrm{n})$ in comparisson to the theoretical prediction of the TALYS code making use of the JLM proton OMP. Deviations between theory and experiment are about $5 \%$ only. 